\title{
Structural Characterization of Hexagonal Braiding Architecture Aided by 3D Printing
}

\author{
Zhengning $\mathrm{Li}^{1,3}$, Ge Chen ${ }^{1}$, Haichen Lyu ${ }^{1}$, Chenwang Yuan ${ }^{2}$ and Frank Ko ${ }^{3}$ \\ ${ }^{1}$ College of Mechanical Engineering, Donghua University, 201620 Shanghai, China \\ ${ }^{2}$ Department of Mechanical Engineering, University of Saskatchewan, S7N 5A9 Saskatoon, Canada \\ ${ }^{3}$ Department of Materials Engineering, University of British Columbia, V6T 1 Z4 Vancouver, Canada
}

\begin{abstract}
Hexagonal braiding method has the advantages of high shape compatibility, interlacing density and high volume fraction. Based on hexagonal braiding method, a hexagonal preform was braided. Then, by following the characteristics of repeatability and concentricity of hexagonal braided preform, the printed geometry structure was got in order to understand and optimize geometric structure to make it more compact like the braided geometric structure. Finally, the unit cells were defined with hexagonal prism to analyze the micro-geometric structure of hexagonal braided preform.
\end{abstract}

\section{Introduction}

Braiding is a traditional textile process that has found wide application for composite manufacturing for the unique characteristics of braided geometry structures such as high damage tolerance, high strength and stiffness, low-cost, reliability and reproducible netshaped preforms. Generally, braided geometry structures are formed by Cartesian braiding and rotary braiding. However, the limitations of these two braiding techniques, such as low packing density, shear deformation and limited preform geometry structure availability constrained further application of braiding geometry structures.

In order to significantly increase the packing density and yarn carrier density of the braiding loom, the concept of hexagonal braiding was developed at AFML (Advanced Fibrous Materials Laboratory) of University of British Columbia. After three generations of optimization, a computer controlled modular hexagonal

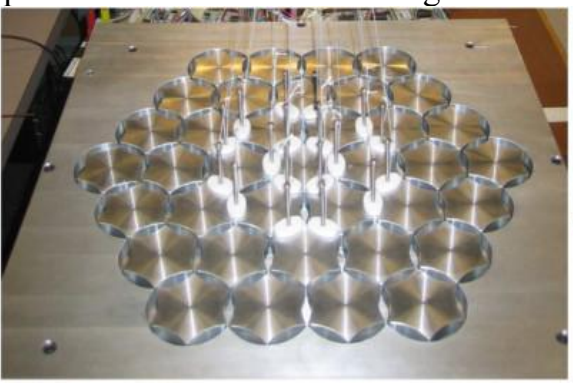

Figure 1. The Hexagonal Machine in UBC.

braiding machine was designed and fabricated jointly by AFML at University of British Columbia (UBC) and ITA at RWTH Aachen University. The hexagonal braiding loom consists of a series of hexagonal horngears and can be easily expanded to variable braiding requirements, as shown in Figure 1 [1].

In hexagonal braiding machine, each hexagonal horngear can drive 6 yarn carriers, comparing to the orthogonal gears in Tuzuki looms with 4 yarn carriers per horngear; The $18 \%$ more yarn carriers can be placed in approximately the same machine footprint area. Due to the universal nature of loom design, the hexagonal braiding allows for a large family of complex 3D polygon shapes such as triangle, hexagon, star, bifurcation, etc., by simply rearranging the yarn carriers as the crosssection shape of preforms and controlling the yarn carriers moving along specific paths, as is shown in Figure 2. More importantly, the horngears in hexagonal loom can rotate $60^{\circ}, 120^{\circ}, 180^{\circ}$ or $240^{\circ}$, which meets the braiding requirement flexibly and the traditional braiding technology, like Cartesian braiding and rotary braiding, cannot do.
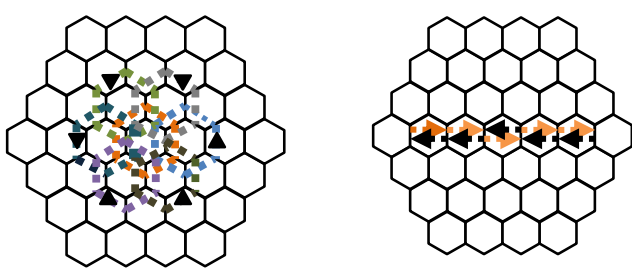

Figure 2. The variable paths of yarn carriers.

The hexagonal geometry structure varies according to the yarn carrier paths and shapes of preforms. For each unique geometry structure, the micro-geometry structure needs to be well defined in order to understand its 
geometry structure and predict the mechanical property. Instead of going through the laborious loom set-up and braiding process, 3D printing, typically Fused Filament Fabrication (FFF), is a promising method to explore various design options and fabrication methods in the formation of complex objects, As shown in Figure 3, after designing the model in CAD software, the model is sliced into a number of layers in slicing software, and then the $3 \mathrm{D}$ printer adds the materials layer by layer to fabricate the designed model. This will significantly reduce capital investment and production cost [5]. Recognizing these benefits 3D printing technology has attracted increasing attention as prototyping equipment for 3D textile preforms, including the topological design. For printed braiding geometry structure, the most excellent characteristic is that the geometry structure is intuitive and can be easy to get, compared to the costly and complex braiding process on braiding looms.

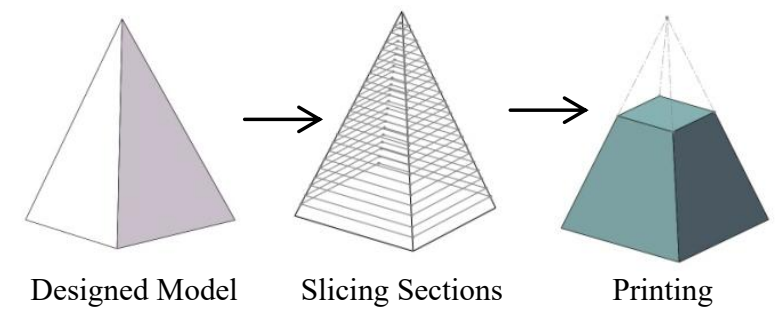

Figure 3. The procedure of $3 \mathrm{D}$ printing

\section{Hexagonal process and parameters}

\subsection{Yarn carriers' motion principles}

As it is mentioned above that hexagonal braiding geometry structures vary according to different movement paths of yarn carriers and yarn carriers' displacement as well, in order to articulate the hexagonal braiding process and hexagonal geometry structure, a kind of solid circular hexagonal geometry structure is created, which contains 30 yarns in the braided preform. In this braiding process, all the 30 yarns are braiding yarns and located at the flanges of seven hexagonal horngears, as shown in Figure 4, there are no axial yarns in this preform. The central horngear 0-1 keeps stationary all the time during braiding process, and the horngears with odd numbers, like 1-1, 1-3, 1-5, rotate clockwise at the same time, likewise, the horngears with even numbers, like 1-2, 1-4, 1-6, rotate counterclockwise at the same time; for each rotation of horngears, the rotation angle is $60^{\circ}$, as shown in Figure 5. These two groups of horngears rotate in turn, and yarn carriers are passed from one horngear to another horngear to lead the yarns intertwining in space, and then the hexagonal preform is produced. The motion sequence and rotation directions of horngears are shown in Table 1. The "+" means horngear rotates clockwise by $60^{\circ}$ and the "-" means the horngear rotates counterclockwise by $60^{\circ}$.
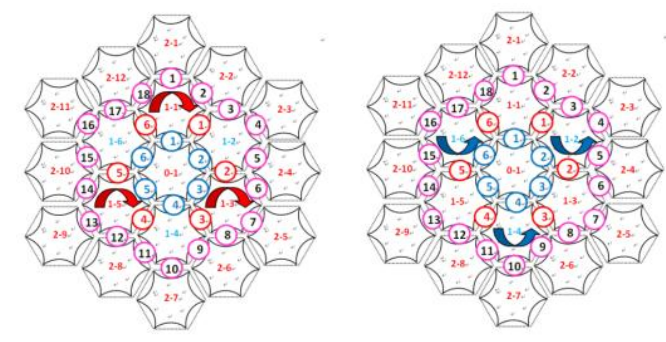

Figure 4. The movement principles of hexagonal horngears

Table 1. The motion sequence and direction of horngears

\begin{tabular}{|c|c|c|}
\hline $\begin{array}{c}\text { Horngear } \\
\text { number }\end{array}$ & Step 1 & Step 2 \\
\hline $1-1$ & + & \\
\hline $1-2$ & & - \\
\hline $1-3$ & + & \\
\hline $1-4$ & & - \\
\hline $1-5$ & + & \\
\hline $1-6$ & & - \\
\hline
\end{tabular}

\subsection{Yarn carriers' path and trajectories}

If the yarn carriers' positions are recorded after every step and the positions of one single yarn carrier are connected with lines, the lines will create a concave polygon path in $\mathrm{X}-\mathrm{Y}$ plane (red lines); for every single yarn, their paths will be like concave polygons (red lines and blue lines), as shown in Figure 6. Following the motion principles mentioned above, every yarn carrier will return their original positions after 30 steps; actually, because of sequence of horngears rotation, the position of every yarn carrier has changed 15 times.

It seems that the paths of 30 yarns preform is the same as maypole braiding, but their braiding processes are totally different, because in maypole braiding process, only half of the intersection points among horngears can be occupied by yarn carriers in order to preventing yarn carrier collision, on the contrary, in hexagonal braiding process, all of the intersection points can be occupied by yarn carriers.

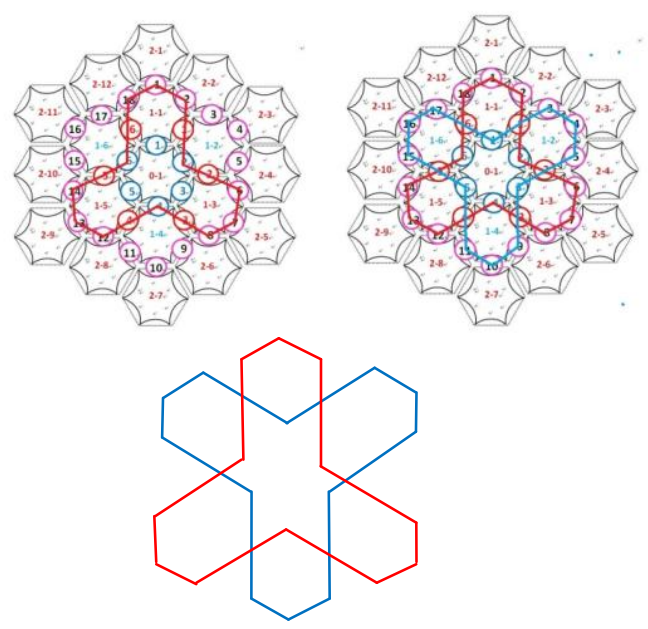

Figure 5. The paths of yarn carriers

During braiding process, braiding yarns are driven by yarn carriers into interlacing in space to produce preforms, and the take-up mechanism will take the preform from the forming plane. If the yarn carriers' positions of every 
step on the braiding bed are connect along the Z-axis, the general trajectories of yarns carriers can be described, the trajectories of yarn carriers can be considered as the yarn trajectories. The yarn carriers' positions are defined as spatial coordinates, and then imported into Matlab to create the trajectories, as show in Figure 6, it seems that the top view of yarn trajectories in Matlab have the same paths as that of yarn carriers in Figure 5. Obviously, the trajectories described in Matlab are not real yarn trajectories, because in braided preform, all the yarns are stretched and cross the preform along the Z-axis in the shortest distance, but as for the yarn trajectories in Matlab, the coordinates are connected by lines, not even curves, so the trajectories should be optimized.
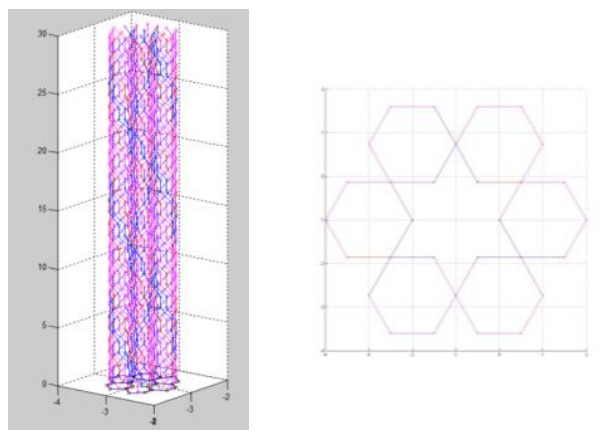

Figure 6. The trajectories of yarns described in Matlab

\section{The establishment of hexagonal geometric structure and optimization by 3D printing}

For the indirect description of yarns' trajectories built in Matlab, the hexagonal geometry structure and microstructure are not easy to understand. If a real 3D model with these trajectories can be got, it would be helpful to clarify and optimize the hexagonal geometry structure. Previous research papers have explored the potential and possibility of printed woven and Cartesian geometry structures, which are based on 3D printing or additive manufacturing, specifically fused filament fabrication (FFF) technology, in that case, the hexagonal geometry structure can be fabricated in the similar way.

\subsection{The 3D model built in CAD}

In order to print $3 \mathrm{D}$ geometry structures, the $3 \mathrm{D} C \mathrm{CAD}$ drawing should be built. In this paper the CAD software, SolidWorks, is used to generate the $3 \mathrm{D}$ model. It is easy to import the coordinates of every yarn into SolidWorks to create yarn curves. By using spline tool, the coordinates are connected by splines and the hexagonal geometry structure has been built, as shown in Figure 8. Compared with the yarn trajectories described in Matlab, the yarn curves in SolidWorks are smoother.

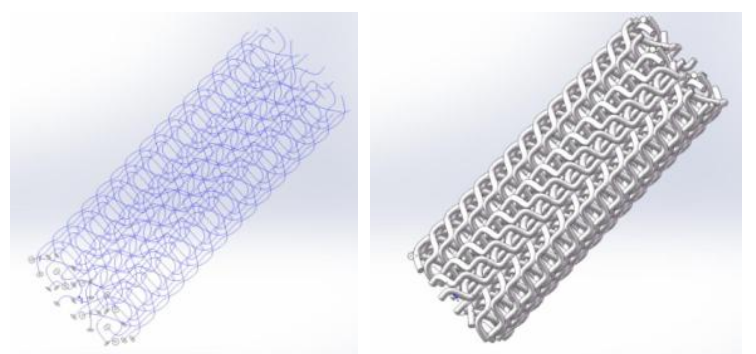

Figure 7. Line drawing and piped structure of yarn trajectories in CAD

A round circle is used to extrude along the spline to create the piped 3D model entity, likewise, all the yarns' entities are built to get the 3D hexagonal piped model, as shown in Figure 7, it seems that all the yarns has the similar tendency along $\mathrm{Z}$-axis in 3D hexagonal piped model. According to the surface of the piped model, yarns have obvious buckling angles at every changing position periodically, the reason for the buckling is that the piped lines go through the positions step by step, not connect the neighbouring coordinates with the shortest line, but it can not be observed in the 3D piped model that whether the internal parts of yarns have the same tendency or not. Hence, the 3D printed model is required.

\subsection{The fabrication of 3D printing model}

Before the fabrication of 3D printed model, the 3D piped model needs to be sliced into layers in the slicing software to generate G-code, which can control the nozzles' movement according to the shape of model's cross-section to extrude and add the molten filament materials on the bed of $3 \mathrm{D}$ printer.

The printing parameters about 3D printer are shown in Table 2. Limited by the maximum printing size of the $3 \mathrm{D}$ printer, the hexagonal geometry structure to be printed was $1 / 3$ part of the all geometry structure, however, the same geometry structure repeats three times during the 30 steps, namely, the printed geometry structure can reveal the yarn trajectories.

Table 2. Parameters to print hexagonal geometry structure

\begin{tabular}{|c|c|}
\hline Parameter & Value \\
\hline Filament Materials & ABS \\
\hline Nozzle Diameter & $400 \mu \mathrm{m}$ \\
\hline Layer Thickness & $210 \mu \mathrm{m}$ \\
\hline Nozzle Temperature & $220^{\circ} \mathrm{C}$ \\
\hline Bedplate Temperature & $105^{\circ} \mathrm{C}$ \\
\hline Printing Speed & $30 \mathrm{~mm} / \mathrm{s}$ \\
\hline
\end{tabular}

Because of the huge gaps among yarns, the support materials are required to add into the model in order to let the printing materials stick together layer by layer during printing process. More importantly, the nozzles' diameter determines printed model's resolution and fidelity, so the yarns diameter in hexagonal geometry structure should be larger than $1000 \mu \mathrm{m}$, namely, $1.00 \mathrm{~mm}$, in which the nozzle's diameter is $400 \mu \mathrm{m}$. For the printed model is 
only used to understand hexagonal geometry structure, not to test the mechanical property, the printing model's infill density is not a requirement and can be set at random.

After the model printing was finished, the support material was removed form the model and the printed hexagonal geometry structure was got, as shown in Figure 8 , the printed hexagonal geometry geometry structure has the same exterior looking as the 3D CAD model, and the yarns interlacing in the center can be observed clearly. However, obviously, the gaps among yarns, longitutionally and radially, are extraordinarily large, it means that the hexagonal geometry geometry structure need to be optimized. Through the tracing yarn (red) in the model, it reveals that every yarn went from the edge.

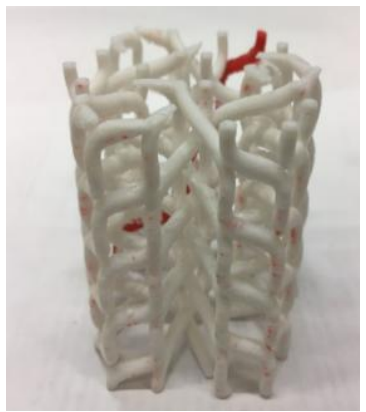

Figure 8. The printed hexagonal geometric structure without optimization

\subsection{The optimization of hexagonal structure}

All the positions of the yarn carriers are concentric, and the yarns are symmetric to the $\mathrm{z}$-axis, the positions are defined as coordinates $\left(\mathrm{x}_{\mathrm{i}}, \mathrm{y}_{\mathrm{i}}, \mathrm{z}_{\mathrm{i}}\right)$, i is positive integer. According to the group thoery, the space lattice which consists of the positions can be converted to new ones by matrixing to make the geometry structure more compact. For the conversion, the conversion matrix is defined as $\mathrm{W}_{\mathrm{i}}$,

$$
\mathrm{W}_{\mathrm{i}}\left[\begin{array}{l}
\mathrm{x} \\
\mathrm{y} \\
\mathrm{z}
\end{array}\right]=\left[\begin{array}{ccc}
\rho_{\mathrm{i} 1} & 0 & 0 \\
0 & \rho_{\mathrm{i} 2} & 0 \\
0 & 0 & \rho_{\mathrm{i} 3}
\end{array}\right]\left[\begin{array}{l}
\mathrm{x} \\
\mathrm{y} \\
\mathrm{z}
\end{array}\right]=\left[\begin{array}{l}
\mathrm{x}^{\prime} \\
\mathrm{y}^{\prime} \\
\mathrm{z}^{\prime}
\end{array}\right]
$$

The conversion coefficients, $\rho_{\mathrm{i} 1}, \rho_{\mathrm{i} 2}, \rho_{\mathrm{i} 3}$, have some relationship with the positions' distance to the center and the jamming condition, and $\rho_{\mathrm{i} 1}, \rho_{\mathrm{i} 2}, \rho_{\mathrm{i} 3}<1$. Let us assume that the positions move along the radius to the center, also made assumptions for geometric geometry structure of hexagonal braiding process:

(1) All the braiding yarns have circular cross section, same linear density and constant fiber packing density;

(2) During the braiding process yarns tension is high enough to make a non-crimp yarn path in hexagonal braiding geometry structure.

So, the coordinates' conversion will follow the relationship below,

$$
\left\{\begin{array}{c}
\mathrm{x}^{\prime}=\rho_{\mathrm{i} 1} \cdot \sqrt{\mathrm{x}^{2}+\mathrm{y}^{2}} \cdot \cos \left(\arctan \left(\frac{\mathrm{y}}{\mathrm{x}}\right)\right) \\
\mathrm{y}^{\prime}=\rho_{\mathrm{i} 2} \cdot \sqrt{\mathrm{x}^{2}+\mathrm{y}^{2}} \cdot \sin \left(\arctan \left(\frac{\mathrm{y}}{\mathrm{x}}\right)\right) \\
\mathrm{z}^{\prime}=\rho_{\mathrm{i} 3} \mathrm{z}
\end{array}\right.
$$

If the rotation angle of yarn jamming is taken into account, function (2) can be revised as,

$\left\{\begin{array}{c}\mathrm{x}^{\prime}=\rho_{\mathrm{i} 1} \cdot \sqrt{\mathrm{x}^{2}+\mathrm{y}^{2}} \cdot \cos \left(\arctan \left(\frac{\mathrm{y}}{\mathrm{x}}\right) \pm \varphi_{\mathrm{i}}\right) \\ \mathrm{y}^{\prime}=\rho_{\mathrm{i} 2} \cdot \sqrt{\mathrm{x}^{2}+\mathrm{y}^{2}} \cdot \sin \left(\arctan \left(\frac{\mathrm{y}}{\mathrm{x}}\right) \pm \varphi_{\mathrm{i}}\right) \\ \mathrm{z}^{\prime}=\rho_{\mathrm{i} 3} \mathrm{z}\end{array}\right.$

Imported the converted coordinate into SolidWorks and use spline to create the trajectories, the piped model was got, as show in Figure 9.
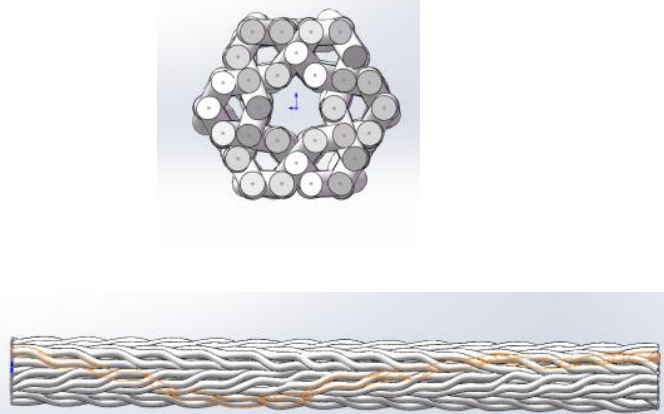

Figure 9. Piped hexagonal geometry geometry structure with optimization

The optimized hexagonal geometry structure has obvious tube in the center of cross-section; for one hand, the yarns deformation and jamming are not considered in this structure, for the other hand, the tubes actually exist in the braided hexagonal preform. As shown in Figure 10, the braided hexagonal preform was cured with resin and cut into pieces; there was a tube in the center of crosssection. Without considering the deformation of yarns, the optimized geometry structure is close to the braided hexagonal preform.

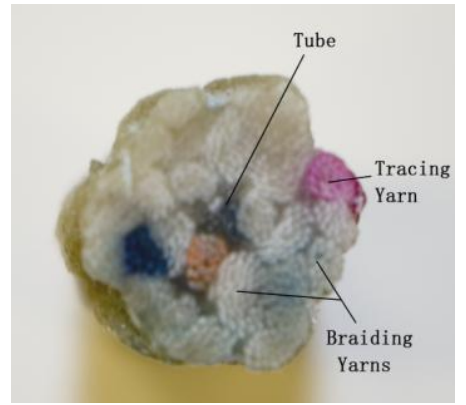

Figure 10. The cross-section of braided hexagonal preform with cured resin

Then, the optimized hexagonal geometry structure was printed by the $3 \mathrm{D}$ printer. Compared with the hexagonal geometry structure without optimization, the optimized hexagonal geometry is more compact, hence it is no need to add support materials during printing to make the geometry structure generated. The printed hexagonal geometry structure with optimization is shown in Figure 11. 


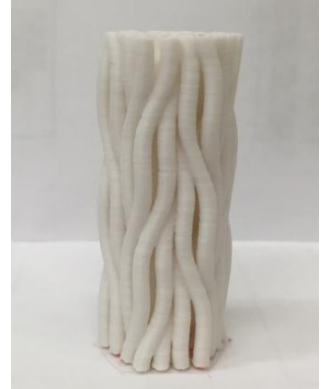

Figure 11. Printed hexagonal geometric structure with optimization

\section{The unit cells of hexagonal structure}

Traditionally, researchers tend to define unit cell geometry to model 3D braided composites. The approach used in geometric modeling of textile geometry structures is to first determine the dimension, shape and fiber geometry structure of the unit cell based on process and structural analysis; using the unit cell geometry identified, the relationship between processing variables and key geometric parameters can readily be established [5]. For traditional braided geometry structures, three types of unit cells, interior cell, corner cell, surface cell, are used to describe the braiding micro-geometry structure. However, for hexagonal geometry structures, because of the repeatability and concentricity, the hexagonal prism can be used to define the unit cell, in that case, there will be two types of unit cells, as show in Figure 12 and Figure 13. In both types of unit cells, they contain 6 bundles of yarns, for one unit cell the yarns have 3 directions, for the other unit cell, the yarns have 6 directions.
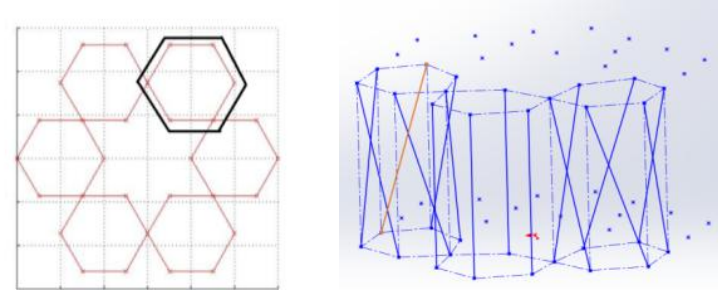

Figure 12. The definition of hexagonal unit cell
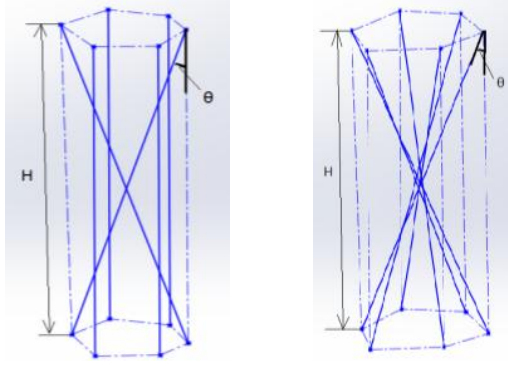

Figure 13. Two types of hexagonal unit cells

\section{Results}

The paper has demonstrated the possibility of using 3D printing technology to aid the geometry structure establishment of hexagonal geometry structure. An optimized hexagonal braiding geometry structure was built through coordinates' conversion and printed geometry structure. Based on micro-geometry structure analysis, two types of unit cells were defined. It reveals that $3 \mathrm{D}$ printing offers us a direct and fast way to establish the hexagonal geometry structure and fabricate the braiding geometry structure. Moreover, in this paper only one kind of circular hexagonal geometry structure was discussed and for the variable hexagonal geometry structures they may have other micro-geometry structure and unit cells, hence, in the future the research emphasis will put on them.

\section{Acknowledgement}

Zhengning $\mathrm{Li}$ acknowledges the support of China Scholarship Council (CSC)

\section{References}

1. Schreiber, F., K. Theelen, and S. Schulte, 18th international conference on composite materials, Jeju Island, Korea (2011)

2. Tsuzuki, M., Kimbara, M., Fukuta, K., Machii, A., US Patent 5,067,525 (1991)

3. Schreiber, F., et al, 17th International Conference on Composite Materials (2009)

4. Quan Z, Larimore Z, Wu A, et al, Composites Science and Technology, E 126, 39-148 (2016)

5. Du G W, Ko F K. Unit Cell Geometry of 3-D Braided Geometry structures. Journal of Reinforced Plastics and Composites, E 12, $752-768$ (1994) 